\title{
Differential effects of changes in mechanical limb properties on physiological and pathological tremor
}

\author{
V HÖMBERG, H HEFTER, K REINERS, H-J FREUND \\ From Neurologische Universitätsklinik, Düsseldorf, Fed. Rep. Germany
}

SUMMARY The effect of changes in mechanical limb properties on the peak frequency of different tremor forms was analysed. Wrist tremor was recorded by an accelerometer fixed to the dorsum of the hand and demodulated surface EMG was recorded from the wrist extensors, while the extended hand was loaded with successively heavier weights. Physiological tremor was characterised by flat EMG spectra and a gradual decrease in tremor peak frequency with increasing load, as would be expected from the properties of a passive spring-mass-system. Also the peak frequency of activated physiological tremor characterised by increased synchronisation between motor units decreased in frequency with increasing loads. EMG spectra showed clear peaks of activity at the various mechanically determined tremor frequencies. In contrast, in two pathological tremor forms, the postural tremor in Parkinsonian patients and essential tremor, peak frequency tended to remain stable irrespective of changes in load. The method therefore allows a simple distinction between physiological and these two pathological tremors.

Physiological tremor is an inevitable byproduct of any motor activity. Clinically tremor is then regarded as abnormal when the resulting movement becomes obvious to the naked eye and tends to interfere with motor performance. Whenever a tremor is recorded it is important to distinguish between two principally different conditions: isometric and isotonic. The difference between these two is that isometric tremor simply reflects those tremor components that are neural in nature. These may result either from the summation properties of asynchronously firing motor units in the narrow frequency range between their onset rates and the discharge frequencies where partial fusion of force ripples occurs, ${ }^{12}$ or from synchronisation between motor units, as in pathological tremors. Such synchronisation mostly takes place at the slowest steady motor unit discharge rates. Even under isometric conditions the synchronised motor unit discharges may excite the muscle spindles thereby bringing the stretch reflex into play. ${ }^{3}$ This becomes particularly obvious in the case of the so called "activated" physiological tremor (anxiety, fatigue, injection of $\beta$-adrenergic drugs) where the amplitude of

Address for reprint requests: Dr V Hömberg, Neurologische Universitätsklinik, Moorenstr. 5, D-4000 Düsseldorf, FRG.

Received 4 January 1985 and in final revised form 19 March 1986. Accepted 24 March 1986 the physiological tremor increases as a result of enhanced synchronisation.

In contrast to isometric, that is force tremor, the tremor recorded under isotonic conditions is largely? dependent on mechanical factors including even the ballistocardiogram. Owing to the wide range of possible mechanical states of the limb with tremor, the mechanical tremor component is subject to considerable modification caused by changes in mechanical limb properties. These alterations are reflected by the changes in amplitude and frequency of tremor.

Under isotonic conditions, the neural component becomes significant only when the motor units are synchronised as in the case of pathological tremor, whereas the physiological isometric (= force) tremor also reflects asynchronous motor unit activity.

This study was designed to examine the effects of changes in mechanical limb properties on the frequency characteristics of physiological isotonic tremor and the interaction between the imposed mechanical factors and the neural synchronisation of pathological tremors. The early investigations by Wertheim-Salomonsen ${ }^{4}$ and Kollaritz ${ }^{5}$ had demonstrated that under physiological conditions isotonic tremor frequency was lowered by adding extra loads to the oscillating hand, pointing to the influence of the mechanical state of the moving limb on its tremor frequency. These results were confirmed and further extended by the use of spectral analysis of isotonic 
limb tremor (for example ref 6). It appeared from these studies that the actual peak frequency of limb tremor under isotonic conditions reflects mass-spring like properties with tremor peak frequency decreasing with increasing inertia or mass. Hence the actual tremor frequency should reflect the resonant frequency of the affected limb and in consequence tremor frequency should be different at different parts of the limb and be altered by adding loads.

We have studied the influence of changing mechanical properties simply by adding weights to the outstretched hand and analysed accelerometer and surface EMG signals to describe the interaction of mechanical and neural factors in various tremor forms. This study does not aim to distinguish to what extent the alteration in tremor frequency is due to changes of mass or of muscle stiffness. It rather focuses on the interaction between the imposed changes of mechanical limb properties and a rhythmic process as it appears in the neural synchronisation in pathological tremors.

The statements: made in this article on tremor parameters all refer to a clearly defined mechanical condition: maintaining constant posture of the hand outstretched against gravity. The results show that in patients with essential or Parkinsonian postural tremor the frequency response to loading the hand is different from that observed in physiological and activated physiological tremor.

\section{Methods}

Subjects Recordings of physiological tremor were made in six normal young subjects (aged 17-33 years, students and hospital staff) without evidence of abnormal tremor. Four patients (aged 22 to 45 years) were classified as having activated physiological tremor because on clinical examination a considerable tremor interfering with every day activities was apparent. Two of these patients had pathological thyroid function, one patient was taking $\beta$-adrenergic drugs and another lithium. From clinical examination there was no evidence of other extrapyramidal signs. In the terminology proposed by Marsden et al ${ }^{7}$ this patient-group would correspond to essential tremor type $\mathbf{I}$.

Fifteen patients (aged 18 to 65 years) were classified as having essential tremor because there was a clinically apparent postural tremor interfering with every day activities. Five of these patients had additional head- and truncal tremor. Most $(n=9)$ had a positive family history and apart from this, there was no evidence of any circumstances known to cause activated physiological tremor. Patients with essential tremor were grouped together and no distinction was made between subgroups (for example type II to III of Marsden et $\mathrm{al}^{7}$.

Recordings were also made in five patients with Parkinson's disease (aged 55 to 70 years). All of them had typical clinical features of Parkinson's disease including prominent tremor at rest. In three of these patients this tremor persisted at the same frequency during constant maintenance of pos- ture as required in our. recording. In two subjects the postural tremor recorded was $1-2 \mathrm{~Hz}$ higher in frequency than their tremor at rest. All were off any medication for at least 3 days preceding the recording.

Procedure Subjects were seated at the side of a laboratory table with their forearms fixed in fully pronated position on a padded mould covering the forearm down to a site $2 \mathrm{~cm}$ proximal to the wrist joint, so that the forearm was supported but not the hand. The fingers were strapped together with adhesive tape keeping all digits in an extended position. The thumb was also fixed in full adduction. This positioning restricted movements mainly to the flexion-extension axis at the wrist joint. Subjects were instructed to keep the hand outstretched over the edge of the mould in a horizontal position with the finger tips pointing to a target mark. All recordings were done under visual control of the subjects and the experimenters ascertained that the hand was maintained in precise horizontal position during the recording. Additional weights from $0 \cdot 1-1.5 \mathrm{~kg}$ could be firmly attached to the dorsum of the hand at a distance of $8 \mathrm{~cm}$ from the wrist joint by means of a light weight plastic surface strapped to the dorsum of the hand with a concave hole in it.

Data recording and analysis A monoaxially sensitive Phillips light weight model PR 9366E/20 accelerometer was strapped with adhesive tape to the middorsum of the hand at a distance of $5 \mathrm{~cm}$ from the wrist joint. Surface EMGelectrodes were attached at a distance of $2 \mathrm{~cm}$ overlying the belly of the extensor carpi radialis (ECR) and extensor digitorum communis (EDC) muscles which under the conditions described were always coactivated. Optimal location of electrodes was achieved by changing electrode position until under isometric conditions a maximal EMG signal was obtained at a constant target force. The EMG signal was amplified (time const. $=10 \mathrm{~ms}$; upper cut-off $3 \mathrm{kHz}$ ), full wave rectified and demodulated by low pass filtering (upper cut-off $30 \mathrm{~Hz}$ ). Before starting tremor recordings it was ensured that no significant EMG activity emerged with passive movements around the wrist to avoid contamination with movement artefacts. In parallel to the demodulated EMG signal the unfiltered EMG was continuously monitored on a storage oscilloscope along with the accelerometer signal. Polaroid photographs were taken from the screen of the storage oscilloscope.

Accelerometer and EMG signals were digitised continuously using a Nicolet Med80 laboratory computer at a sampling rate of $60 \mathrm{~Hz}$ and stored on magnetic discs. Using the fast Fourier transform algorithm, power spectral density functions were computed based on analysis epochs of $4 \cdot 2$ seconds for accelerometer and EMG signals. This yielded a resolution of $0.125 \mathrm{~Hz}$ in the frequency domain. Spectra of eight consecutive $4 \cdot 2$ second epochs were condensed to one average spectrum representing a total duration of about 34 seconds for analysis. From these spectra the following parameters were extracted: (a) peak frequency, defined as maximal frequency between 2 and $17 \mathrm{~Hz}$; (b) peak power; (c) total spectral power between 2 and $17 \mathrm{~Hz}$; (d) peak power ratio defined as the ratio between power in a $1 \mathrm{~Hz}$ band around the peak and total spectral power. The higher the peak power ratio, the more prominent is the tremor peak with respect to the remainder of the spectrum. The eight $\mathbf{4 \cdot 2}$ seconds epochs as well as an average spectrum comprising 
the whole duration of analysis at a given load level were plotted for the frequency band between 2 and $17 \mathrm{~Hz}$. To achieve optimal resolution of frequency composition, in this paper spectral plots are presented as autoscaled spectra keeping peak amplitude at a constant plot height. They therefore do not contain absolute peak amplitude information. Absolute peak power values of the accelerometer recordings are given numerically beside each spectrum in the figures.

\section{Results}

The effect of loading on physiological and activated physiological tremors

Figure 1 presents typical spectra of accelerometer and demodulated EMG signals of non-activated physiological tremor at successively heavier loads. Tremor peak frequencies in the unloaded condition varied between 8.0 and $10.2 \mathrm{~Hz}$. There is a clear shift of dominant tremor rate to lower frequencies with increasing weights. The ongoing EMG activity does not show obvious bursts reflecting synchronisation at smaller loads. Correspondingly the average spectra of the demodulated EMG reveal no consistent peaks at loads up to 200 grams. At higher loads however, clearer peaks become apparent in the EMG spectra, at frequencies of around $10 \mathrm{~Hz}$. These peaks in the EMG spectra occur at considerably higher frequencies than the peaks of the accelerometer readings. The group mean of total tremor power was $3.37 \mathrm{~g}^{2} \times 10^{-3}$ (range 1.1 to 11.6 ) in the unloaded condition. Tremor power monotonically decreased with increasing loadings (see fig 7A). Peak power ratios were in the range of $0 \cdot 10-0.30$ for accelerometer and in the range of $0 \cdot 20-0.44$ for EMG signals.

In this group of patients with activated physiological tremor, tremor peak frequency in the unloaded condition varied between $6 \mathrm{~Hz}$ and $8 \cdot 3 \mathrm{~Hz}$. Total tremor power was considerably higher than in the group with physiological tremor (group mean = $38.0 \mathrm{~g}^{2} \times 10^{-3}$, range $=10 \cdot 2$ to 64.8 ) in the unloaded condition. Figure 2 shows typical spectra of a patient with activated physiological tremor: similar to the findings in non-activated physiological tremor the dominant tremor rate decreases with increasing load. But the concomitant EMG spectra show distinct peaks that also decrease in frequency in parallel to the change of mechanical resonant frequency. Only at highest loads a second peak appears in the EMG spectra at frequencies above $10 \mathrm{~Hz}$, which is also reflected by a second peak in the accelerometer spectrum. In comparison with the group of subjects with non-activated physiological tremor peak power ratios are higher for both accelerometer (range 0.4-0.8) and especially EMG spectra (range $0.6-0.9$ ) in the unloaded condition. The decay in tremor amplitudes with increasing loads is similar in both activated and non-activated physiological tremor (fig 7B). It therefore appears, that dominant tremor rate in both

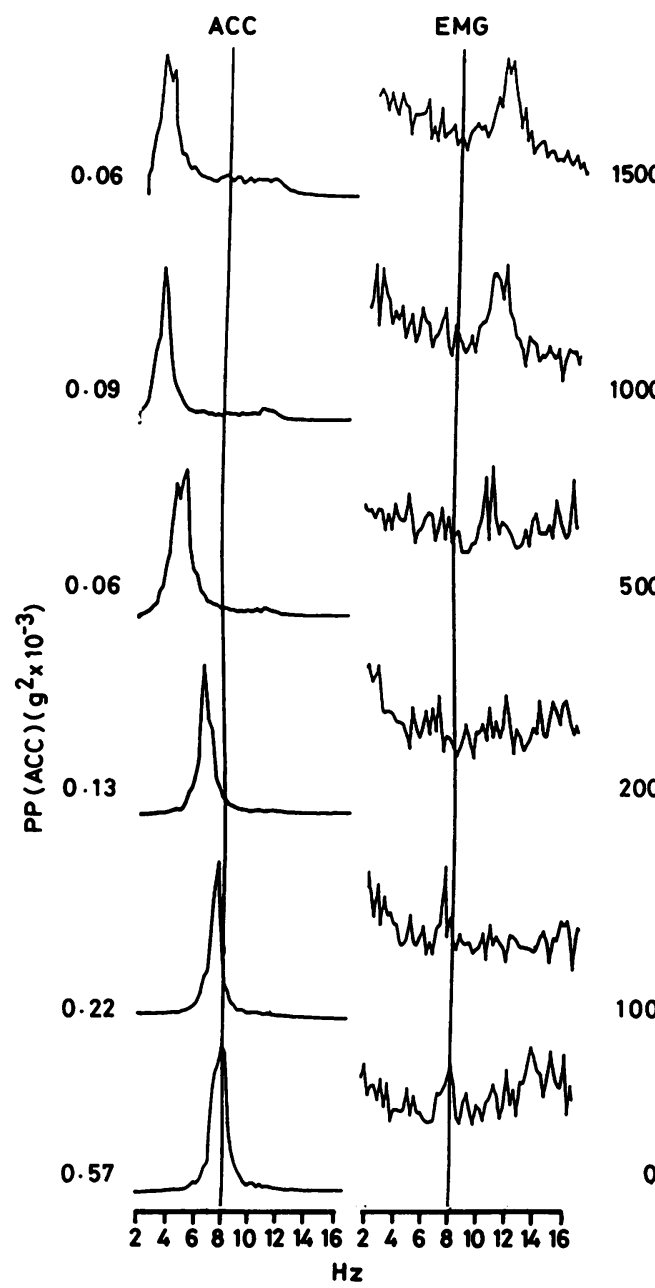

Fig 1 Accelerometer (left panel) and wrist extensor EMG spectra (right panel) of wrist tremor recordings in a 28 year old male subject with physiological tremor. Each spectrum represents the average of eight consecutive analysis epochs of $4 \cdot 2 \mathrm{~s}=34 \mathrm{~s}$ total analysis time for each loading. With increasing loads (from bottom to top) tremor peak frequency gradually decreases. Note that tremor amplitudes are autoscaled in this and all subsequent accelerometer spectra so that each peak is plotted at constant height to achieve optimal display of frequency shifts. The absolute peak power values of the accelerometer recordings $(P P(A C C))\left(\right.$ in $\left.g^{2} \times 10^{-3}\right)$ are indicated numerically aside the accelerometer spectra. The EMG spectra at lower load levels do not contain significant peaks. Only with high loads spectral peaks become apparent in the EMG spectra reflecting synchronisation of motor unit activity at frequencies above $10 \mathrm{~Hz}$. 
physiological and activated physiological tremors is determined by the mechanical resonant frequency. The main difference between activated and nonactivated physiological tremors is an increased synchronisation in activated physiological tremor leading to higher tremor amplitudes. The rate at which this synchronisation occurs follows the

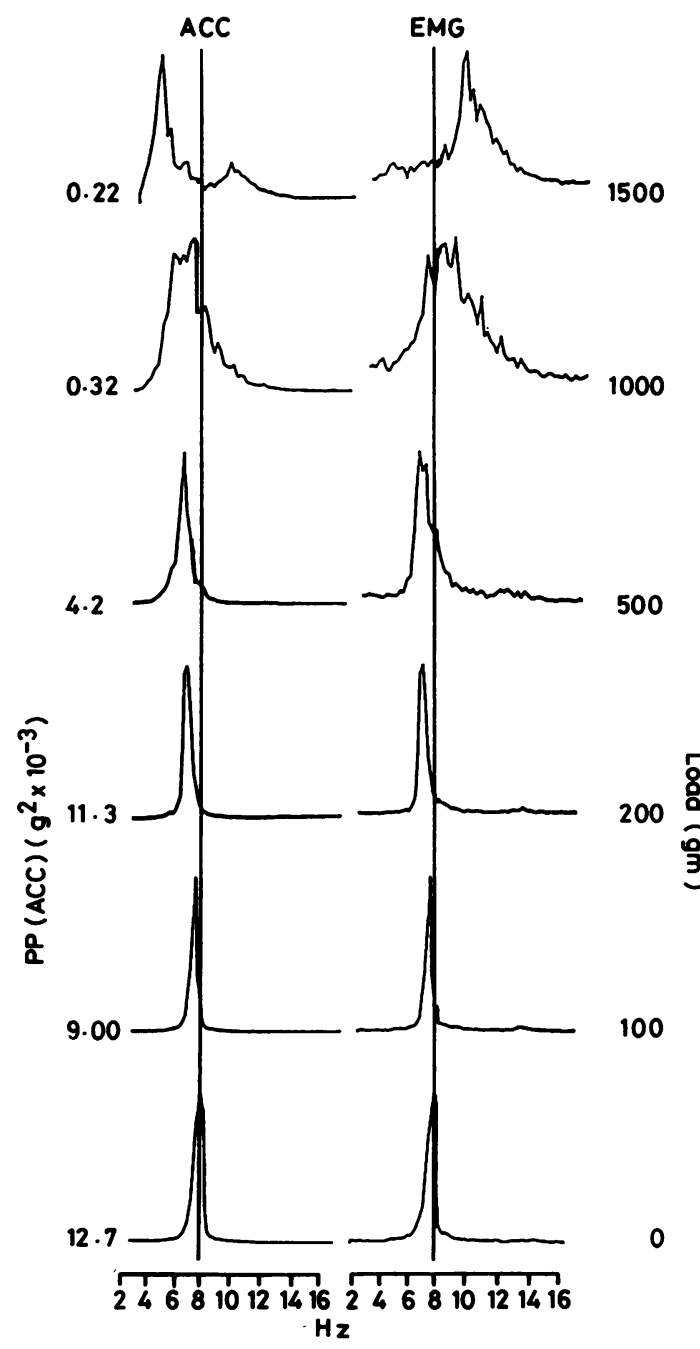

Fig 2 Accelerometer (left panel) and wrist extensor EMG (right panel) spectra in a 30 year old male patient with activated physiological tremor due to hyperthyroidism. Both accelerometer and EMG spectra show sharp peaks, which gradually decrease in frequency with increasing loads. Only at highest load levels the EMG peak shifts to a high frequency. In this patient tremor peak power values (PP (Acc)) are much higher than in the subject with non-activated physiological tremor in fig 1 . resonant frequency determined by the mechanical conditions of the trembling hand.

Both physiological and activated physiological tremors show a divergence of accelerometer and EMG peak frequencies at higher loads: at very high loadings, which require increased force output, a synchronised neural activity at around $10 \mathrm{~Hz}$ becomes apparent. This is clearly distinct from the mechanically influenced synchronisation-frequencies apparent at lower loading levels and is most marked in the records with activated physiological tremor. This tremor component is not modified by loading. It is likely to be neural in origin as it is evident predominantly in the EMG spectra, whereas the peak frequencies of the accelerometer recordings reflect the larger amplitude oscillations at the lower mechanical resonant frequencies induced by these high loadings.

The effect of loading on pathological tremors

Tremor frequencies in patients with essential tremor in the unloaded condition varied between 5.7 and

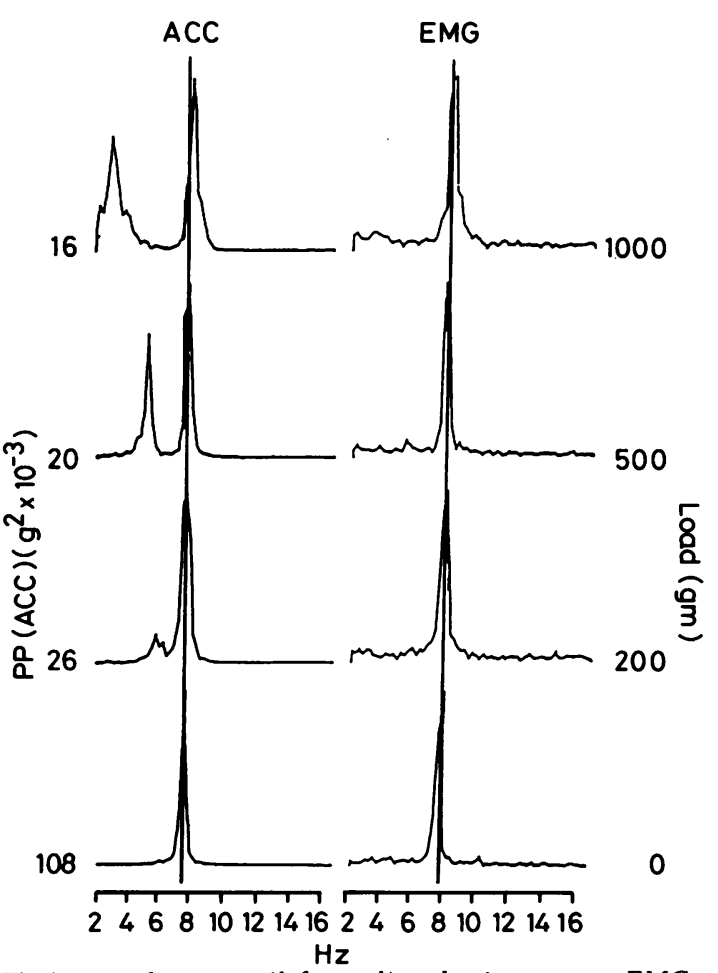

Fig 3 Accelerometer (left panel) and wrist extensor EMG (right panel) spectra of a 50 year old male with essential tremor. Both accelerometer and EMG spectra show sharp peaks at around $7 \mathrm{~Hz}$ which remain fairly constant with increasing loads. In the accelerometer spectra secondary peaks appear which gradually decrease in frequency concomitant with lowering of mechanical resonant frequency. 
$8.6 \mathrm{~Hz}$. Total tremor power was higher than in the groups with physiological tremors (group mean $=$ $195 \mathrm{~g}^{2} \times 10^{-3}$, range $=45$ to 604 in the unloaded condition.) Figure 3 illustrates spectra of a patient with essential tremor. In contrast to the load dependent changes observed in physiological and activated physiological tremors, peak frequency remains stable between 7 and $8 \mathrm{~Hz}$ irrespective of the changing in mechanical properties of the trembling hand. The demodulated EMG spectra show a clear distinct peak exactly at this frequency. Ongoing EMG correspondingly reveals a clear grouping of bursts of activity at $7-8 \mathrm{~Hz}$ unaffected by adding various loads with a stable pattern of simultaneous activity in agonist (wrist extensor) and antagonist (wrist flexor) muscles (fig 4B). In contrast the EMG pattern of a subject with physiological tremor (fig 4A) lacks obvious grouping at both low and high loadings.

Simultaneous activation of agonist and antagonist muscles is known to be characteristic of essential tremor, ${ }^{8}$ but some patients in this group may also show reciprocal activity in antagonistic muscles. ${ }^{9}$ The accelerometer spectra shown in fig 3 contain a second peak, which varies with changes in the mechanical limb properties but is not paralleled by corresponding peaks in the EMG spectra. This second peak occurs at (a)

$1.0 \mathrm{~kg}$

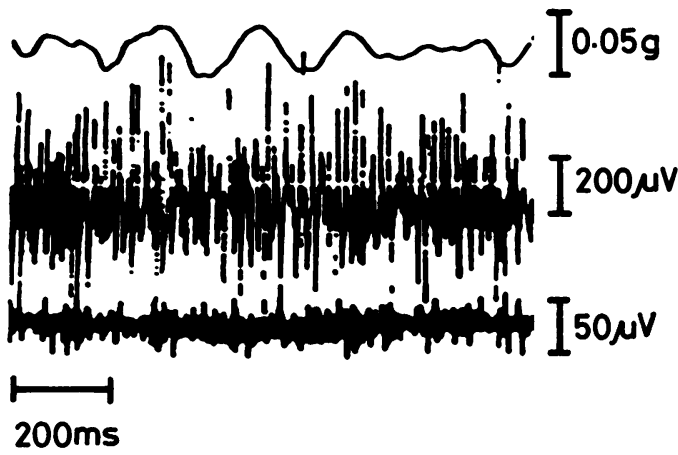

$0.1 \mathrm{~kg}$

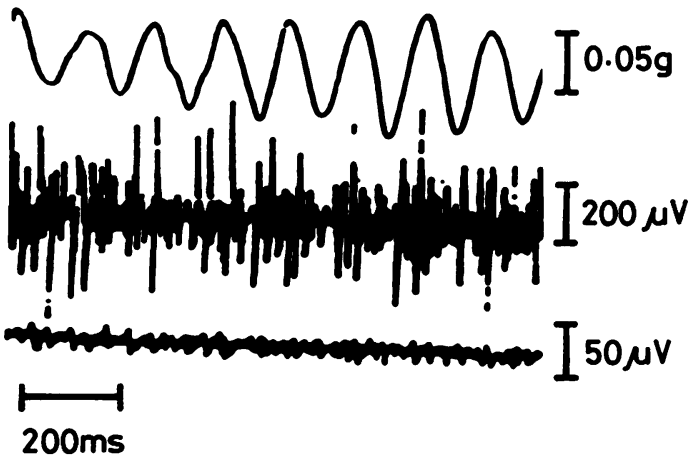

(b)

$1.0 \mathrm{~kg}$

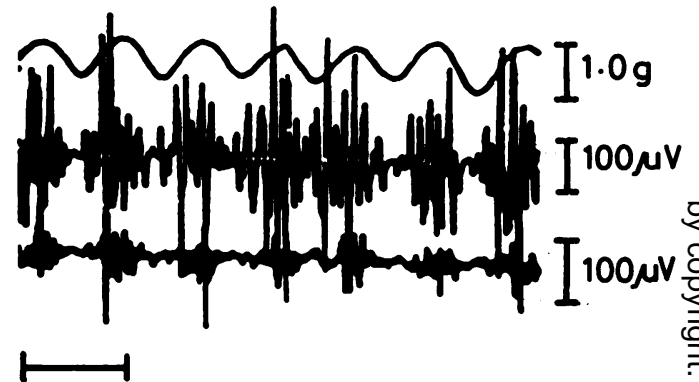

$200 \mathrm{~ms}$

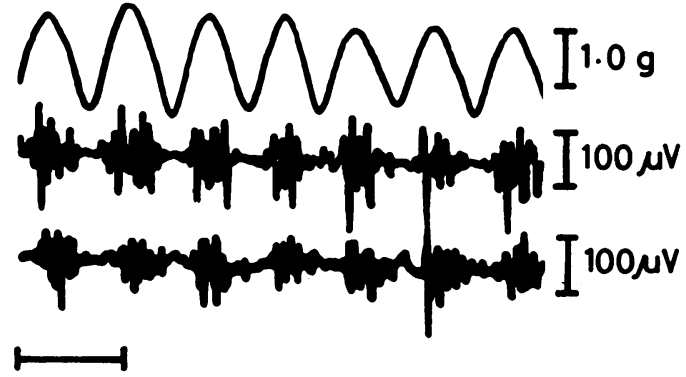

$200 \mathrm{~ms}$

Fig 4 Accelerometer (upper trace), wrist extensor (middle trace) and wrist flexor raw EMG (lower trace) in a subject with physiological tremor $(A)$ and a patient with essential tremor $(B)$ with low loading $(0 \cdot 1 \mathrm{~kg}$, lower part) and high loading $(1.0 \mathrm{~kg}$, upper part $)$. Note change in tremor frequency with increasing load in the subject with physiological tremor and lack of bursts of activity in the EMG traces. Extensor EMG increases in amplitude with higher load. In contrast, in the essential tremor patient tremor frequency remains stable and the EMG shows a constant burst repetition rate with coactivation of agonist and antagonist muscles. 
the same frequencies as the mechanically induced peaks seen in the spectra of subjects with physiological tremor. Peak power ratios in essential tremor patients ranged between 0.62 and 0.92 for accelerometer and between 0.54 and 0.84 for EMG signals. Total tremor power at higher loadings decreased to a much lower extent and less monotonically than in the groups with physiological and activated physiological tremors (fig 7C).

Figure 5 illustrates the load-independent constancy of tremor frequency in a patient with Parkinsonian tremor $(5 \mathrm{~Hz})$ at rest, which persisted during constant maintenance of posture. Tremor frequencies in the unloaded condition in this group ranged between 4.5 and $6.9 \mathrm{~Hz}$. Total tremor power in the group of Par-

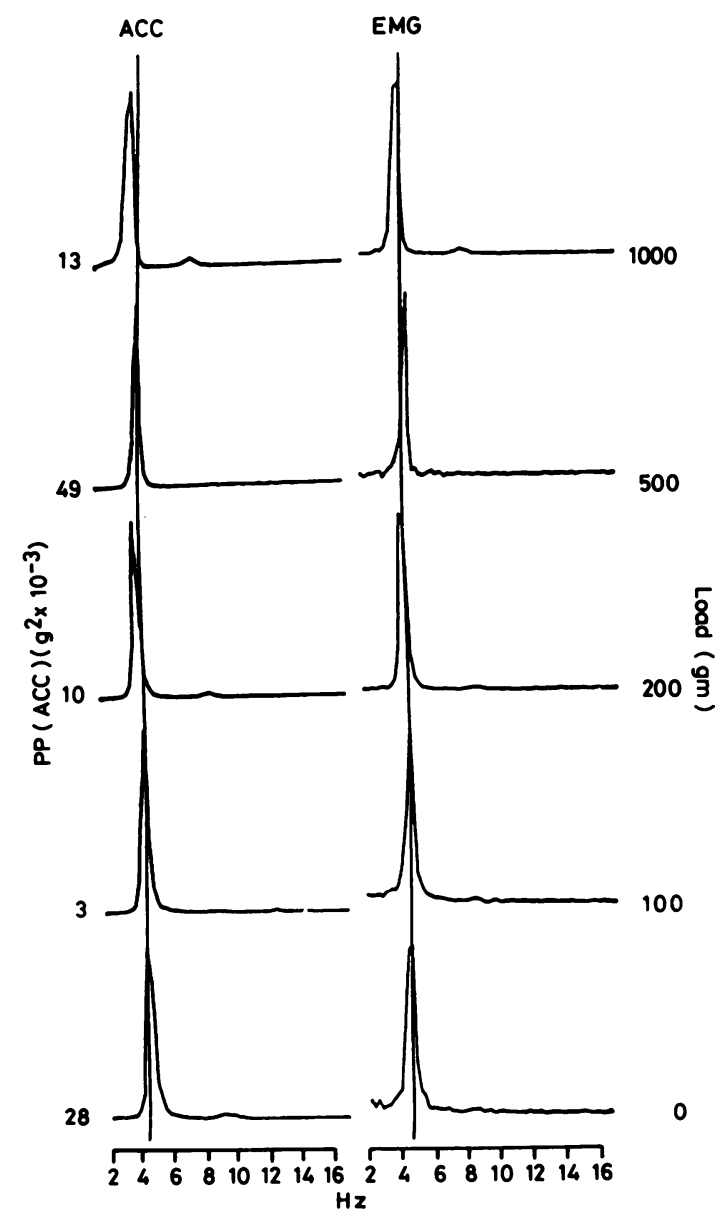

Fig 5 Accelerometer (left panel) and wrist extensor EMG spectra (right panel) in a 61 year old Parkinsonian patient. Both accelerometer and EMG spectra show sharp peaks remaining constant in frequency irrespective of changes in load. kinsonian patients was lower than in the essential tremor patients (group mean $=93.3 \mathrm{~g}^{2} \times 10^{-3}$, range $=27-279$ ). As in essential tremor peak frequency is not affected by changes in loading and is paralleled by a high degree of EMG synchronisation as revealed by sharp peaks in the demodulated EMG spectra reflecting bursts in the ongoing EMG. The EMG showed a reciprocal pattern of agonist and antagonist activity. This constancy of dominant tremor rate was similarly observed in all Parkinsonian patients irrespective of whether the recorded postural tremor was at the same or at higher frequency compared to their resting tremor. Total tremor power only slightly decreased with increasing amounts of load (fig 7D). Peak power ratios in the Parkinsonian patients ranged between 0.56 and 0.96 for accelerometer and between 0.43 and 0.89 for EMG signals.

Figure 6 summarises the accelerometer peak frequency vs load relationship for groups of subjects with physiological tremor, activated physiological tremor and pathological tremors. Whereas both types of physiological tremor show a similar decrease of peak frequency with increasing loads all Parkinsonian and essential tremors show a nearly constant peak frequency independent from changes in the mechanical state of the limb.

In some of the patients with essential tremors we noted some increase in dominant tremor rate with high amounts of added mass along with the concomitant increase in muscle tension. This was always accompanied by corresponding spectral changes in the EMG pointing to a neural origin. This increase in tremor peak frequencies at very high loadings appears to be similar to the occurrence of a higher frequency neural synchronisation at highest load levels, which we also observed in physiological tremors.

Figure 7 summarises the group statistical results of load-changes on total tremor power in the different groups. Whereas in both physiological and activated physiological tremor, spectral power is considerably lowered with higher loadings, this effect is less pronounced in both pathological tremor groups. In physiological and activated physiological tremor the amplitude reduction follows a monotonic trend. In the pathological tremor groups however relative power maxima may occur at intermediate load levels. These maxima show considerable interindividual variation and hence do not reach statistical significance. Since the loading was always applied in a fixed increasing sequence systematic effects related to muscular fatigue (increased tremor amplitude after previous higher loads) or anxiety caused by the unfamiliar recording situation (increased tremor amplitude at beginning of the session) are unlikely to cause the observed deviance from a monotonic trend. These rather seem to reflect random tremor amplitude 
(a) Physiological tremor

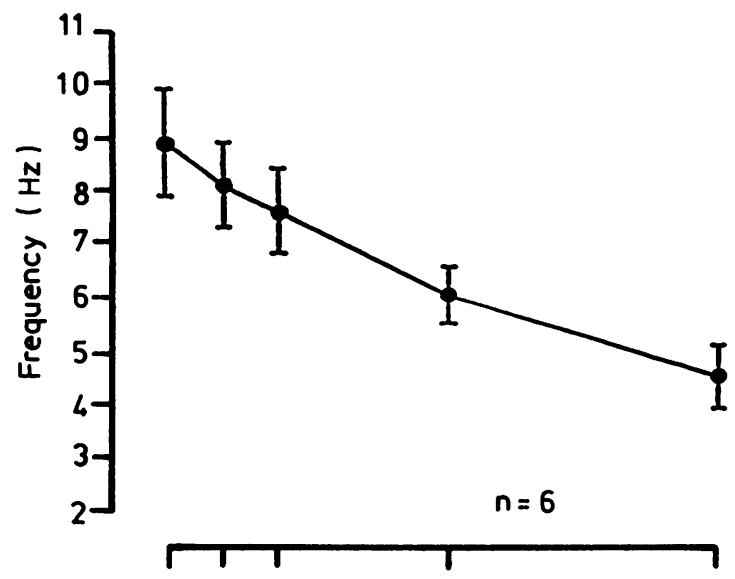

C) Essential tremor

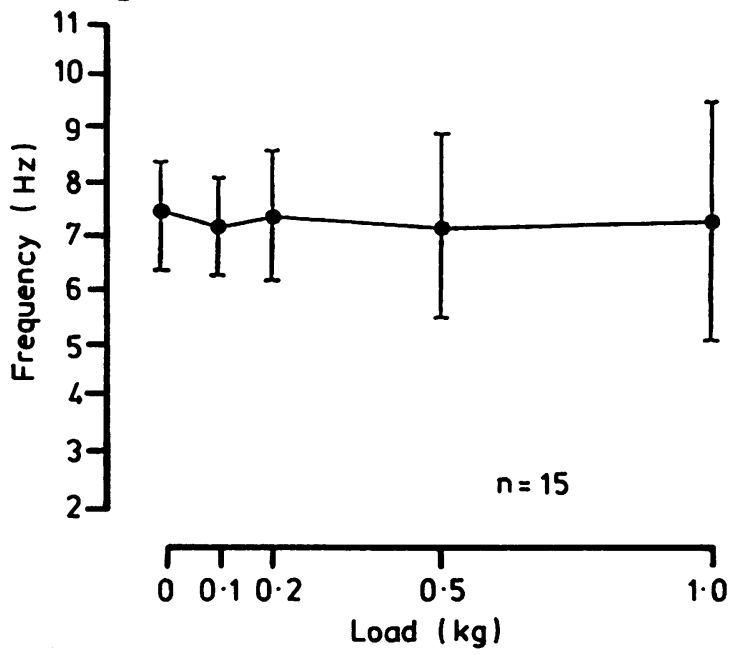

b) Activated physiological tremor

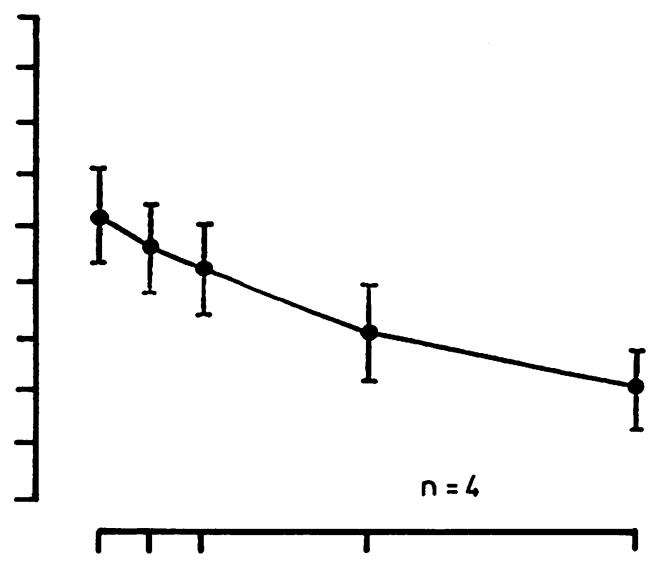

(d) Parkinsonian tremor

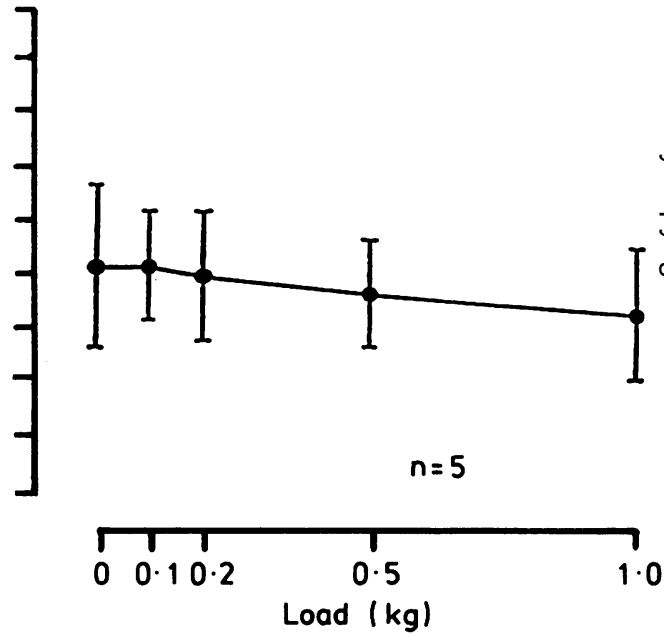

Fig 6 Group means and standard deviations of accelerometer recorded tremor peak freqiency with changes in load in groups of subjects with physiological $(A)$, activated physiological $(B)$, essential $(C)$ and Parkinsonian (D) tremors.

variations not related to the experimental variables most marked in the pathological tremors. Therefore the mass-related mechanical damping of the pathological tremors appears less consistent and pronounced.

\section{Discussion}

In this study we investigated the relative contributions of mechanical and neural factors in the generation of physiological and pathological tremor under isotonic conditions. The mechanical limb properties were altered by adding various extra loads.
This was done in subjects with physiological tremor who showed no significant synchronisation between motor units, in subjects with activated physiological tremor who showed some motor unit synchronisation and in patients with pathological tremors characterised by strong synchronisation. The principal result is a consistent qualitative finding: whereas both physiological and activated physiological tremors show a clear decrease in peak frequency with increasing load, the dominant rates of both essential and Parkinsonian tremors are not affected by mechanical changes. 
(a) Physiological tremor

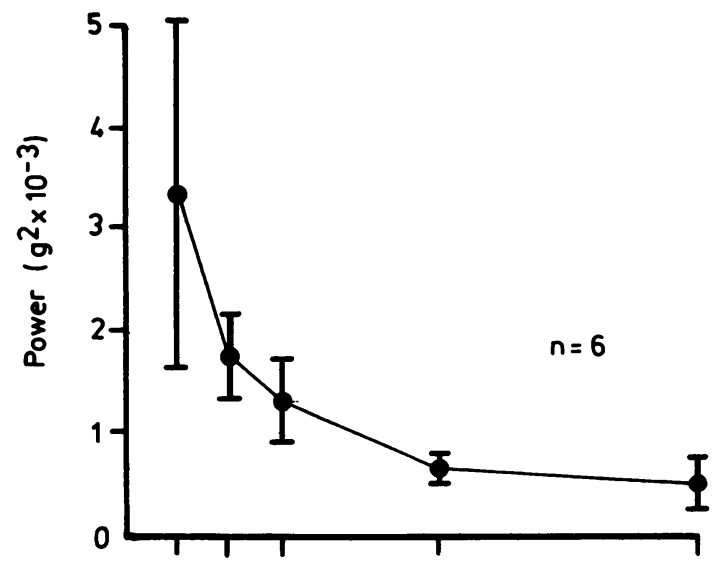

(c) Essential tremor

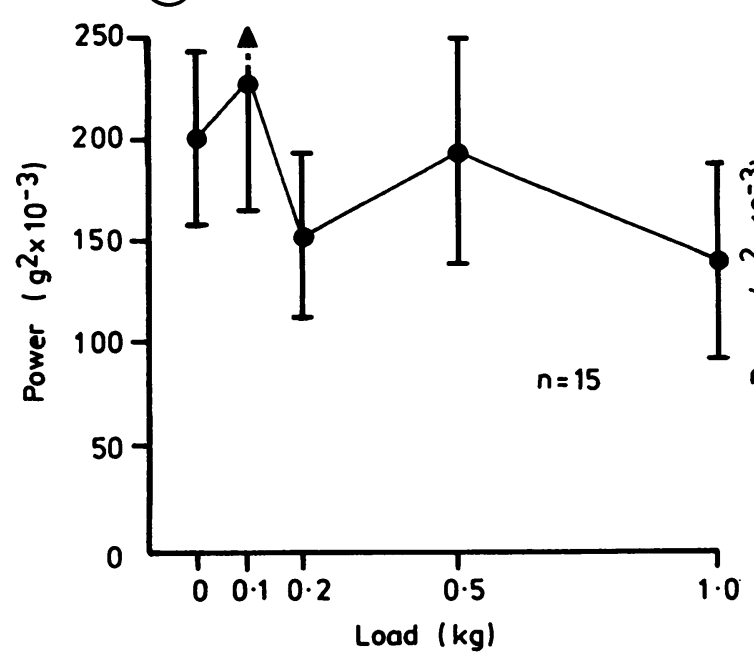

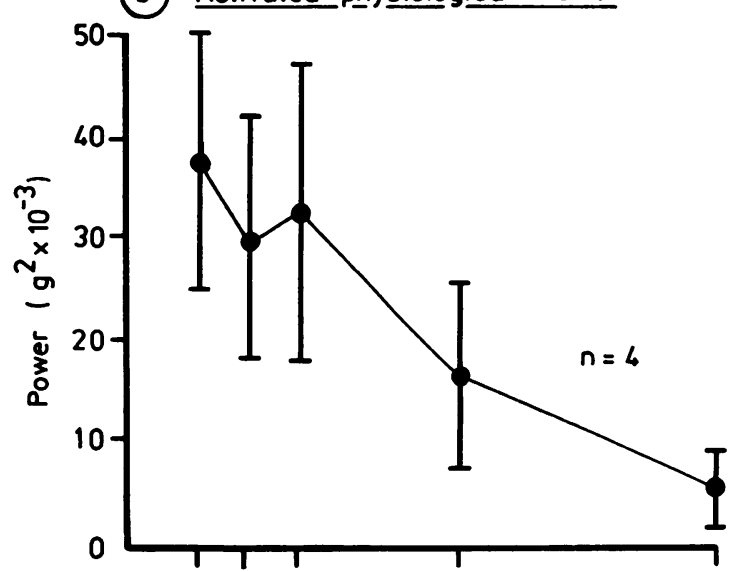

(d) Parkinsonian tremor

Fig 7 Group means and standard errors of total accelerometer recorded tremor power in the frequency band between 2 and 17 $\mathrm{Hz}$ at different loads for the groups with physiological $(A)$, activated physiological $(B)$, essential $(C)$ and Parkinsonian (D) tremors.

Three principal patterns of interaction of mechanical and neural factors were observed:

(1) In physiological tremor peak frequency was always dependent on the mechanical resonant frequency and there was no prominent peaking in the demodulated EMG spectra or grouping of activity (that is, bursts) in the ongoing EMG.

(2) In "activated" physiological tremor peak frequency was also determined by the mechanical resonant frequency. However, EMG spectra showed clear peaks reflecting neural synchronisation at frequencies corresponding to the peaks in the accelerometer spectra. Occasionally in both physiological and activated physiological tremor a separate neural tremor com- ponent with frequencies at around $10 \mathrm{~Hz}$ tended to appear in the EMG spectra at high load levels. This tremor component was independent from changes in mechanical resonant frequency.

(3) In both essential and Parkinsonian tremors the dominant tremor rate was not affected by changes in mass and the EMG showed burst repetition rates at a constant frequency reflected by corresponding sharp peaks in the EMG spectra irrespective of changes in mechanical resonant frequency. The prominent peaks in the EMG and in the accelerometer spectra always occurred at the same frequencies.

Our procedure to change the mechanical state of the limb by adding various weights to the horizontally 

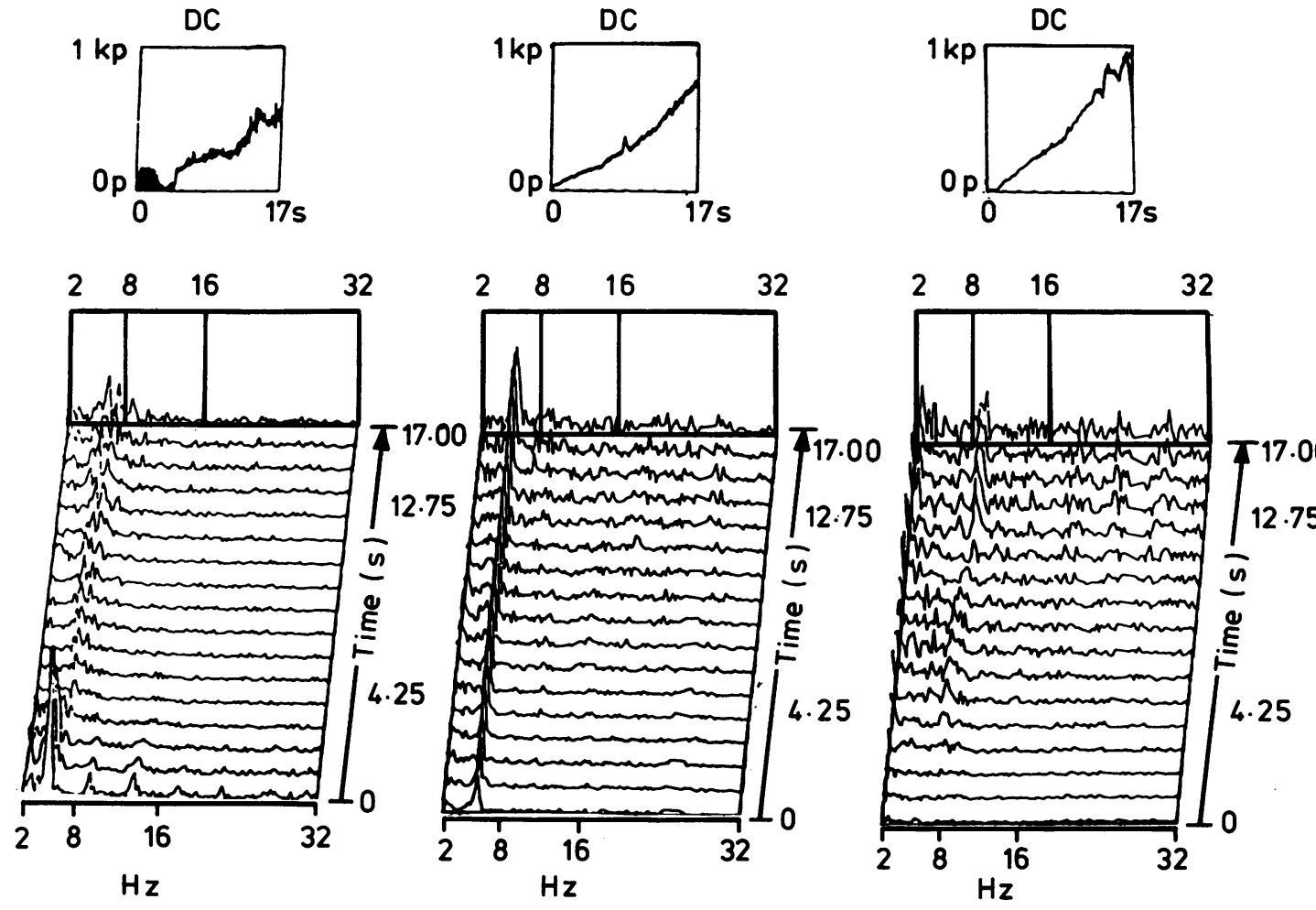

Fig 8 Consecutive partially overlapping power spectra (based on $4 \cdot 25$ s epochs) calculated from isometric force tremor recordings of the index finger in two patients with Parkinson's disease. Each series represents time course of slow ramp increases in force as indicated by the DC-coupled force recordings in the inserts at the top of each panel. In a Parkinsonian patient with typical low frequency pathological tremor at rest (left panel) this tremor is blocked after starting the contraction and is replaced by a physiological force tremor characterised by less sharp, more variable peaks at higher frequencies. In another Parkinsonian patient with severe tremor at rest, this low frequency tremor prevails during execution of the contraction (middle panel). After levodopa treatment in the same patient this pathological tremor is abolished by physiological force tremor (right panel). For methodical details see ref 2.

outstretched hand inevitably changes both mass and muscle stiffness. Thus from the experimental design it is not possible to distinguish the extent to which the resulting change in resonant frequency is due to either of these factors. This is, however, not relevant for the distinction between normal and abnormal tremor in respect of the different interaction between mechanical and neural factors in these two groups.

\section{Physiological tremor}

The observation that the frequency of physiological tremor under isotonic conditions depends on the mechanical resonant frequency confirms earlier investigations. ${ }^{610}$ In this condition there were no apparent bursts in the ongoing EMG and no prominent peaks in the power spectra of the demodulated EMG. This is in agreement with observations by Hagbarth and Young ${ }^{3}$ who also noted a lack of rhythmicity in EMG signals in non-activated physiological tremor proper.

\section{Activated physiological tremor}

The main difference between recordings in this group as compared with subjects with non-activated physiological tremor were larger tremor amplitudes and higher relative peak power ratios reflected by sharper peaks in accelerometer and EMG recordings. As in physiological tremor, peak tremor frequency shifted with changes in mechanical resonant frequency. The fact that this was paralleled also by changes in the peaks of the EMG spectra points to an increase in gain of the stretch reflex loop leading to increased synchronisation of motoneuron pool outflow phaselocked to the stretching parts of the movement cycle. The periodicity of this oscillation is determined by the mechanical resonant frequency. The observed shifts 
in spectral peaks with increasing loads thus point to a peripheral modulation of the synchronisation of motoneuron pool activity supporting the idea of a reflex mechanism mediating this enhanced motoneuron pool synchronisation. Following the observations by Hagbarth and Young ${ }^{3}$ of enhanced grouping of primary muscle spindle afferents in activated physiological tremor, a sensitisation of spindle input has been assumed as the most likely mechanism for this increase in gain of the spinal reflex loop. ${ }^{11}$ The data indicate that motor unit synchronisation at the same part of the body can be induced over a wide range of different frequencies. This observation emphasises that the frequency of a rhythmical oscillation in the skeletomotor apparatus does not provide information about loop times: reflex supported, activated physiological tremor can be deliberately induced at various frequencies just by changing mechanical properties of one particular part of a limb, where the loop-times can be considered to remain constant. Changes in tremor frequencies with alteration of mechanical conditions have also been obtained for human elbow tremor when subjects with compliant loading exert constant force against springs of different stiffness: both tremor and EMG spectra showed a shift towards higher frequencies with increasing spring stiffness. ${ }^{11}$ This is in agreement with mathematical models examining the effects of loop times of multiple reflex pathways in interaction with their relative gain ${ }^{12}$ on the resulting oscillations. Then frequency is dependent on loop times as well as on the distribution of gains within these multiple loops. Therefore any interpretations linking higher frequency oscillations with shorter loops and low frequency oscillations with longer reflex loops always have to ascertain identical mechanical conditions and unchanged gains when multiple reflex loops participate in generation of the oscillations studied. Within the range of mechanical changes and resulting resonant frequencies examined here, the rate of neural synchronisation mechanism involved in activated physiological tremor follows changes in the mechanical resonant frequencies.

In both subjects with physiological and activated physiological tremor a neural synchronisation around $10 \mathrm{~Hz}$ could be observed at higher loads documented by circumscribed peaks in the EMG spectra. This tremor component was clearly distinct from the mechanically determined activated physiological tremor, which decreased in frequency at higher loads. This neural tremor of higher frequency resembles the $8-12 \mathrm{~Hz}$ tremor as observed by Elble and coworkers, ${ }^{13}$ who recently described the occurrence of such a tremor component in $30 \%$ of their normals, being present in parallel to a mechanically sensitive tremor: in contrast to the mechanically determined tremor peak, this $8-12 \mathrm{~Hz}$ tremor could not be reset by mechanical perturbations. Furthermore this $8-12 \mathrm{~Hz}$ tremor corresponds in peak frequency to the isometrically recorded force tremor emerging during prolonged isometric contractions at maximal forces. ${ }^{2}$

\section{Pathological tremors}

In contrast to physiological and activated physiological tremor, the dominant rate of the pathological postural tremors could not be influenced by adding loads to the trembling limb. Similar findings distinguishing physiological and essential tremors by their different response to mechanical changes have been published recently by Elble. ${ }^{14}$

It appears that the dominant rate of these pathological tremors cannot be determined by mechanical alterations, pointing to a central origin. Thus there is a clear cut difference between essential tremor and activated physiological tremor. This leads us not to include the latter as type I of the essential tremors as proposed by the classification of Marsden et al. ${ }^{7}$

In the two pathological tremors examined in this study the periodicity of the central oscillator dominates the mechanical influence. These may still cause a secondary oscillation of lower amplitude visible as a secondary peak in the tremor spectrum. Our findings point to a central generator with spontaneous oscillating discharges not influenced by peripheral inputs (such as changes in the mechanical state of the limb) as the cause of both pathological tremor-forms studied here. They do not support the view that pathological tremors are due to self-sustaining oscillations of peripheral reflex loops (for example ref 15). Nevertheless the question remains whether there is a possible interaction of afferent input and the central oscillator possibly coupled to this input. In a reflex study Lee and Stein ${ }^{16}$ using sudden torque pertubations of ongoing tremor activity have shown that essential tremors could be resynchronised by such a procedure whereas Parkinsonian tremor could not. This reveals that change of peripheral input have some access to the rhythmical oscillator of essential tremor but not to the generators of Parkinsonian tremor.

The contention of a non-reflex-mediated central origin of Parkinsonian tremor is also corroborated by microneurographic studies ${ }^{1718}$ describing recordings made from primary spindle afferents in patients with Parkinsonian tremor. These studies revealed a pattern of alpha-gamma-coactivation similar to voluntary alternating movements and pointed to a non-spindle reflex mediated central origin of the rhythmical tremor movement in Parkinson's disease. In contradiction to earlier studies ${ }^{1920}$ recently Rack and Ross $^{21}$ observed some minor frequency changes with changes in inertia in the wrist tremor of Parkinson's 
disease. The neural origin of this change was documented by changes in the repetition rate of EMGbursts. Their results however showed considerable intra- and inter-individual variation which makes it difficult to judge the significance of these findings.

How far the results obtained on two pathological tremor forms here apply to other pathological tremors remains to be shown. Vilis and Hore ${ }^{22}$ used chronic cryoprobe implants in the cerebellar nuclei of non-human primates to induce reversible cerebellar intention tremor. They noted that the frequency of cerebellar tremor covaried with changes in the mechanical state of the limb in a way predictable from passive spring-load properties. This would imply a stronger modification of cerebellar tremor by peripheral input than seen in our patients with essential tremor or Parkinson's disease.

The results of this study were obtained in a particular standardised mechanical situation. It is, however, a characteristic feature of the pathological tremors that they vary under different postural conditions. For this reason it is clinical practice to distinguish between tremor at rest, action tremor, postural tremor, intention tremor and other special tremor forms.

Parkinsonian tremor is usually a tremor at rest which is diminished during increasing muscular effort. In contrast essential tremor is most pronounced during motor activity no matter if this is stationary such as maintaining a constant posture (postural tremor) or if it is non-stationary motor activity (action or kinetic tremor). In more severe cases of essential tremor this tremor may also appear at rest and conversely in advanced Parkinsonian cases the resting tremor will persist during the maintenance of posture. This variability of tremors with regard to amplitude and frequency in different innervational states is illustrated in fig 8 for two patients with Parkinsonian tremor. Isometric recording of force tremor (for methodological details see ref 2) during voluntary contraction shows different changes of tremor. In a patient with typical Parkinsonian tremor at rest (left panel) the low frequency tremor of his index finger is immediately blocked after he starts to press against the force transducer and is transformed to or substituted by a less synchronised force tremor which resembles physiological force tremor. In another Parkinsonian patient the prevailing low frequency tremor at rest (middle panel) persists throughout the contraction. After treatment of this patient with levodopa this high amplitude low frequency tremor is replaced by a physiological force tremor (right panel). For this reason we feel it is not justified to classify tremors primarily according to their preferential postural appearance.

Posture, state of muscle activity, emotional and mechanical factors are all determinants for the amplitude-frequency profile of tremor as revealed by spectral analysis. The reasons why and the mechanisms how they exert their differential effects are barely understood. The information obtained from this study (in addition to its diagnostic value) is that the rate of pathological tremors with strongest motor unit synchronisation seems not to be influenced by gross changes in the resonant properties of the trembling limb.

This work was supported by grants from the Deutsche Forschungsgemeinschaft (SFB 200, B3). Preliminary data of this study have been presented at the 7th European Neuroscience Congress Hamburg 1983.

\section{References}

1 Allum JHJ, Dietz V, Freund H-J. Neuronal mechanisms underlying physiological tremor. $J$ Neurophysiol 1978;41:557-71.

2 Hömberg V, Reiners K, Hefter H, Freund HJ. The muscle activity spectrum: spectral analysis of muscle force as an estimator of overall motor unit activity. Electroencephalogr Clin Neurophysiol 1986;63:209-22.

3 Hagbarth KE, Young RR. Participation of the Stretch Reflex in Human Physiological Tremor. Brain 1979;102:509-26.

4 Wertheim-Salomonson JKA. Beitrag zum Studium des Zitterns. Dtsch Z Nervenheilk 1897;10:243-72.

5 Kollaritz J. Über das Zittern. Disch Z Nervenheilk 1910;38:438-79.

6 Stiles RN, Randall JE. Mechanical factors in human tremor frequency. J Appl Physiol 1967;23:324-30.

7 Marsden CD, Obeso J, Rothwell JC. Benign essential tremor is not a single entity. In: Yahr, M.D. (ed.) Current Concepts in Parkinson's Disease. Excerpta Medica, Amsterdam, 1983:31-46.

8 Shahani BT, Young RR. Physiological and pharmacological aids in the differential diagnosis of tremor J Neurol Neurosurg Psychiatry 1976;39:772-83.

9 Sabra AF, Hallett M. Action tremor with alternating activity in antagonist muscles. Neurology 1984;34: $151-6$.

10 Hamoen AM, Über das Wesen des physiologischen Tremors der Hand. Psychiat Neurol Neurochir 1962;65:103-8.

11 Matthews PBC, Muier RB. Comparison of electromyogram spectra with force spectra during human elbow tremor. J Physiol (London) 1980;302:427-41.

12 Oguztöreli MN, Stein RB. The effects of multiple reflex pathways on the oscillations in neuro-muscular systems. J Mathemat Biol 1976;3:87-101.

13 Elble RJ, Adelman J, Higgins C. Interaction between stretch reflex and 8-12 Hz component of physiologic tremor. Soc Neurosci Abstr 1985;11:302.4.

14 Elble RJ. Physiologic and essential tremor. Neurology 1986;36:225-31. 
15 Stiles RN, Pozos RS. A mechanical reflex oscillator hypothesis for Parkinsonian hand tremor. J Appl Physiol 1976;40:990-8.

16 Lee RG, Stein RB. Resetting of Tremor by Mechanical Perturbations: A Comparison of Essential Tremor and Parkinsonian Tremor. Ann Neurol 1981;10:523-31.

17 Hagbarth KE, Wallin G, Löfstedt L, Aquilonius S-M. Muscle spindle activity in alternating tremor of Parkinsonism and in clonus. $J$ Neurol Neurosurg Psychiatry 1975;38:636-41.

18 Wallin G, Hagbarth K-E. Muscle spindly activity in man during voluntary alternating movements, Parkinsonian tremor and clonus. Progr Clin Neurophysiol
1978;5:150-9.

19 Rack PMH. Mechanical and reflex factors in human tremor. In: Desmedt JE. (ed.) Physiological Tremor, Pathological Tremors and Clonus. Prog Clin Neurophysiol, (Basel) 1978;5:17-27.

20 Walsh EG. Beats produced between a rhythmic applied force in the resting tremor of Parkinsonism. $J$ Neurol Neurosurg Psychiatr 1980;42:89-94.

21 Rack PMH, Ross HF. The role of reflexes in the resting tremor of Parkinson's disease. Brain 1986;109:115-41.

22 Vilis T. Hore J. Effects of changes in mechanical state of limb on cerebellar intention tremor. $J$ Neurophysiol 1977;40:1214-224. 